\title{
Fixed point results under c-distance in tvs-cone metric spaces
}

\author{
Momčilo Đorđević ${ }^{1}$, Dragan Đorić 2 , Zoran Kadelburg ${ }^{3}$, Stojan Radenović ${ }^{4 *}$ and Dragoljub Spasić ${ }^{1}$
}

\footnotetext{
* Correspondence: radens@beotel. net

${ }^{4}$ Faculty of Mechanical Engineering, University of Belgrade, Kraljice Marije 16, 11120 Beograd, Serbia Full list of author information is available at the end of the article
}

\author{
Abstract \\ Fixed point and common fixed point results for mappings in tvs-cone metric spaces \\ (with the underlying cone which is not normal) under contractive conditions \\ expressed in the terms of c-distance are obtained. Respective results concerning \\ mappings without periodic points are also deduced. Examples are given to \\ distinguish these results from the known ones. \\ Mathematics Subject Classification (2010) \\ $47 \mathrm{H} 10,54 \mathrm{H} 25$
}

Keywords: Tvs-cone metric space, c-Distance, Common fixed point

\section{Introduction}

Cone metric spaces were considered by Huang and Zhang in [1], who reintroduced the concept which has been known since the middle of 20th century (see, e.g., [2-4]). Topological vector space-valued version of these spaces was treated in [5-13]; see also [14] for a survey of fixed point results in these spaces.

Fixed point theorems in metric spaces with the so-called $w$-distance were obtained for the first time by Kada et al. in [15] where nonconvex minimization problems were treated. Further results were given, e.g., in [16-18]. Cone metric version of this notion (usually called a $c$-distance) was used, e.g., in $[19,20]$.

In this paper, we consider fixed point and common fixed point results for mappings in tvs-cone metric spaces (with the underlying cone which is not normal) under contractive conditions expressed in the terms of $c$-distance. Respective results concerning mappings without periodic points are also deduced. Examples are given to distinguish these results from the known ones.

\section{Preliminaries}

Let $E$ be a real Hausdorff topological vector space ( $t v s$ for short) with the zero vector $\theta$. A proper nonempty and closed subset $P$ of $E$ is called a cone if $P+P \subset P, \lambda P \subset P$ for $\lambda \geq 0$ and $P \cap(-P)=\{\theta\}$. We shall always assume that the cone $P$ has a nonempty interior int $P$ (such cones are called solid).

Each cone $P$ induces a partial order $\leqslant$ on $E$ by $x \leqslant y \Leftrightarrow y-x \in P$. $x \pi y$ will stand for $(x \leqslant y$ and $x \neq y)$, while $x \ll y$ will stand for $y-x \in$ int $P$. The pair $(E, P)$ is an ordered topological vector space.

\section{SpringerOpen ${ }^{\circ}$}

(c) 2011 Đorđevićć et al; licensee Springer. This is an Open Access article distributed under the terms of the Creative Commons Attribution License (http://creativecommons.org/licenses/by/2.0), which permits unrestricted use, distribution, and reproduction in any medium, provided the original work is properly cited. 
For a pair of elements $x, y$ in $E$ such that $x \leqslant y$, put $[x, y]=\{z \in E: x \leqslant z \leqslant y\}$. A subset $A$ of $E$ is said to be order-convex if $[x, y] \subset A$, whenever $x, y \in A$ and $x \leqslant y$.

Ordered topological vector space $(E, P)$ is order-convex if it has a base of neighborhoods of $\theta$ consisting of order-convex subsets. In this case, the cone $P$ is said to be normal. If $E$ is a normed space, this condition means that the unit ball is order-convex, which is equivalent to the condition that there is a number $k$ such that $x, y \in E$ and 0 $\leqslant x \leqslant y$ implies that $\|x\| \leq k|| y \|$. A proof of the following assertion can be found, e.g., in [2].

Theorem 1 If the underlying cone of an ordered tvs is solid and normal, then such tvs must be an ordered normed space.

Note that completions of cone metric spaces in the case of nonnormal underlying cone were treated in [21].

From [1,5-7], we give the following

Definition 1 Let $X$ be a nonempty set and $(E, P)$ an ordered $t v s$. A function $d: X \times$ $X \rightarrow E$ is called a tvs-cone metric and $(X, d)$ is called a tvs-cone metric space if the following conditions hold:

(c1) $\theta \leqslant d(x, y)$ for all $x, y \in X$ and $d(x, y)=\theta$ if and only if $x=y$;

(c2) $d(x, y)=d(y, x)$ for all $x, y \in X$;

(c3) $d(x, z) \leqslant d(x, y)+d(y, z)$ for all $x, y, z \in X$.

Taking into account Theorem 1, proper generalizations when passing from normvalued cone metric spaces of [1] to tvs-cone metric spaces can be obtained only in the case of nonnormal cones. We shall make use of the following properties:

$\left(\mathrm{p}_{1}\right)$ If $u, v, w \in E, u \leqslant v$ and $v \ll w$ then $u \ll w$.

$\left(\mathrm{p}_{2}\right)$ If $u \in E$ and $\theta \leqslant u \ll c$ for each $c \in$ int $P$ then $u=\theta$.

$\left(\mathrm{p}_{3}\right)$ If $u_{n}, v_{n}, u, v \in E, \theta \leqslant u_{n} \leqslant v_{n}$ for each $n \in \mathbb{N}$, and $u_{n} \rightarrow u, v_{n} \rightarrow v(n \rightarrow \infty)$, then $\theta \leqslant u \leqslant v$.

$\left(\mathrm{p}_{4}\right)$ If $x_{n}, x \in X, u_{n} \in E, d\left(x_{n}, x\right) \leqslant u_{n}$ and $u_{n} \rightarrow \theta(n \rightarrow \infty)$, then $x_{n} \rightarrow x(n \rightarrow \infty)$.

$\left(\mathrm{p}_{5}\right)$ If $u \leqslant \lambda u$, where $u \in P$ and $0 \leq \lambda<1$, then $u=\theta$.

$\left(\mathrm{p}_{6}\right)$ If $c \gg \theta$ and $u_{n} \in E, u_{n} \rightarrow \theta(n \rightarrow \infty)$, then there exists $n_{0}$ such that $u_{n} \ll c$ for all $n \geq n_{0}$.

In the sequel, $E$ will always denote a topological vector space, with the zero vector $\theta$ and with order relation $\preccurlyeq$, generated by a solid cone $P$. For notions such as convergent and Cauchy sequences, completeness, continuity etc. in (tvs)-cone metric spaces, we refer to $[1,7,14]$ and references therein.

Kada et al. [15] introduced the notion of $w$-distance in metric spaces and proved some fixed point results using this notion (see also [16-18]). Cho et al. [19] transferred it to the setting of cone metric spaces (see also [20]).

Definition 2 [19] Let $(X, d)$ be a tvs-cone metric space. A function $q: X \times X \rightarrow E$ is called a c-distance in $X$ if:

(q1) $\theta \leqslant q(x, y)$ for all $x, y \in X$;

(q2) $q(x, z) \leqslant q(x, y)+q(y, z)$ for all $x, y, z \in X$;

(q3) If a sequence $\left\{y_{n}\right\}$ in $X$ converges to a point $y \in X$, and for some $x \in X$ and $u=$ $u_{x} \in P, q\left(x, y_{n}\right) \leqslant u$ holds for each $n \in \mathbb{N}$, then $q(x, y) \leqslant u$;

(q4) For each $c \in E$ with $\theta \ll c$, there exists $e \in E$ with $\theta \leqslant e$, such that $q(z, x) \ll e$ and $q(z, y) \ll e$ implies $d(x, y) \ll c$. 
Each $w$-distance $q$ in a metric space $(X, d)$ (in the sense of [15]) is a $c$-distance in the tvs-cone metric space $(X, d)$ (with $E=\mathbb{R}$ and $P=[0,+\infty)$ ). Indeed, only property (q3) has to be checked. Let $y_{n} \in X, y_{n} \rightarrow y$ in the cone metric $d(n \rightarrow \infty)$, and let $q\left(x, y_{n}\right) \leq$ $u_{x} \in[0,+\infty$ ). Since $q$ is (as a $w$-distance) lower semi-continuous, we have that $q(x, y)$ $\leq \lim \inf _{n \rightarrow \infty} q\left(x, y_{n}\right) \leq \lim _{\inf _{n \rightarrow \infty}} u_{x}=u_{x}$, i.e., $q(x, y) \leq u_{x}$ holds true.

The first two of the following examples are variations of [[19], Examples 2.7, 2.8], adjusted to the case of a tvs-cone metric.

Example 1 Let $(X, d)$ be a tvs-cone metric space such that the metric $d(\cdot, \cdot)$ is a continuous function in second variable. Then, $q(x, y)=d(x, y)$ is a $c$-distance. Indeed, only property (q3) is nontrivial and it follows from $q\left(x, y_{n}\right)=d\left(x, y_{n}\right) \leqslant u$, passing to the limit when $n \rightarrow \infty$ and using continuity of $d$.

Example 2 Let $(X, d)$ be a tvs-cone metric space, and let $u \in X$ be fixed. Then, $q(x$, $y)=d(u, y)$ defines a $c$-distance on $X$. Indeed, (q1) and (q3) are clear. (q2) follows from $q(x, z)=d(u, z) \leqslant d(u, y)+d(u, z)=q(x, y)+q(y, z)$. Finally, (q4) is obtained by taking $e=c / 2$.

Example 3 Consider the Banach space $E=C[0,1]$ of real-valued continuous functions with the max-norm and ordered by the cone $P=\{f \in E: f(t) \geq 0$ for $t \in[0,1]\}$. This cone is normal in the Banach-space topology on $E$. Let $\tau^{*}$ be the strongest locally convex topology on the vector space $E$. Then, the cone $P$ is solid, but it is not normal in the topology $\tau^{*}$. Indeed, if this were the case, Theorem 1 would imply that the topology $\tau^{*}$ is normed, which is impossible since an infinite dimensional space with the strongest locally convex topology cannot be metrizable (see, e.g., [14]).

Let now $X=[0,+\infty)$ and $d: X \times X \rightarrow\left(E, \tau^{*}\right)$ be defined by $d(x, y)(t)=|x-y| \phi(t)$ for a fixed element $\phi \in P$. Then, $(X, d)$ is a tvs-cone metric space which is not a cone metric space in the sense of [1]. We can introduce two $c$-distances on this space:

$$
q_{1}(x, y)(t)=x \cdot \varphi(t), \quad \text { and } \quad q_{2}(x, y)(t)=y \cdot \varphi(t) .
$$

They are the examples of $c$-distances in tvs-cone metric spaces which are not $c$-distances in cone metric spaces of $[19,20]$.

These examples show, among other things, that for a $c$-distance $q$ :

1. $q(x, y)=q(y, x)$ does not necessarily hold for all $x, y \in X$;

2. $q(x, y)=\theta$ is not necessarily equivalent to $x=y$.

\section{Results}

\subsection{Fixed point and common fixed point results under c-distance}

We will call a sequence $\left\{u_{n}\right\}$ in $P$ a $c$-sequence if for each $c \gg \theta$ there exists $n_{0} \in \mathrm{N}$ such that $u_{n} \ll c$ for $n \geq n_{0}$. It is easy to show that if $\left\{u_{n}\right\}$ and $\left\{v_{n}\right\}$ are $c$-sequences in $E$ and $\alpha, \beta>0$, then $\left\{\alpha u_{n}+\beta v_{n}\right\}$ is a $c$-sequence.

Note that in the case that the cone $P$ is normal, a sequence in $E$ is a $c$-sequence iff it is a $\theta$-sequence (see property $\left(\mathrm{p}_{6}\right)$ ). However, when the cone is not normal, a $c$ sequence need not be a $\theta$-sequence $($ see $[7,14])$. Also, from [7], we know that the cone metric $d$ need not be a continuous function.

The following lemma is a tvs-cone metric version of lemmas from [15,19].

Lemma 1 Let $(X, d)$ be a tvs-cone metric space and let $q$ be a c-distance on $X$. Let $\left\{x_{n}\right\}$ and $\left\{y_{n}\right\}$ be sequences in $\times$ and $x, y, z \in X$. Suppose that $\left\{u_{n}\right\}$ and $\left\{v_{n}\right\}$ are csequences in $P$. Then the following hold: 
(1) If $q\left(x_{n}, y\right) \leqslant u_{n}$ and $q\left(x_{n}, z\right) \leqslant v_{n}$ for $n \in \mathbb{N}$, then $y=z$. In particular, if $q(x, y)=\theta$ and $q(x, z)=\theta$, then $y=z$.

(2) If $q\left(x_{n}, y_{n}\right) \leqslant u_{n}$ and $q\left(x_{n}, z\right) \leqslant v_{n}$ for $n \in \mathbb{N}$, then $\left\{y_{n}\right\}$ converges to $z$.

(3) If $q\left(x_{n}, x_{m}\right) \leqslant u_{n}$ for $m>n>n_{0}$, then $\left\{x_{n}\right\}$ is a Cauchy sequence in $X$.

(4) If $q\left(y, x_{n}\right) \leqslant u_{n}$ for $n \in \mathbb{N}$, then $\left\{x_{n}\right\}$ is a Cauchy sequence in $X$.

Proof We will prove assertions (1) and (2). Proofs of the other two are similar.

(1) In order to prove that $y=z$, according to $\left(\mathrm{p}_{2}\right)$, it is enough to show that $d(y, z)$ $\ll c$ for each $c \gg \theta$. For the given $c$ choose $e \gg \theta$ such that property (q4) is satisfied. Choose then $n_{0} \in \mathbb{N}$ such that $u_{n} \ll e$ and $v_{n} \ll e$ for $n \geq n_{0}$. Then, by property $\left(\mathrm{p}_{1}\right)$, we get that $q\left(x_{n}, y\right) \ll e$ and $q\left(x_{n}, z\right) \ll e$ and (q4) imply that $d(y, z) \ll c$.

(2) Let again $c \ll \theta$ be arbitrary and choose a corresponding $e \gg \theta$ satisfying property (q4). If $n_{0} \in \mathrm{N}$ is such that $u_{n} \ll e$ and $v_{n} \ll e$ for $n \geq n_{0}$, then $\left(\mathrm{p}_{1}\right)$ implies that $q$ $\left(x_{n}, y_{n}\right) \ll e$ and $q\left(x_{n}, z\right) \ll e$ for $n \geq n_{0}$. Then, by (q4), $d\left(y_{n}, z\right) \ll c$ and $y_{n} \rightarrow z(n \rightarrow$ $\infty)$.

Our first result is the following theorem of Hardy-Rogers type.

Theorem 2 Let $(X, d)$ be a complete tvs-cone metric space and let $q$ be a c-distance on $X$. Suppose that a continuous self-map $f: X \rightarrow X$ satisfies the following two conditions:

$$
\begin{aligned}
& q(f x, f y) \preccurlyeq A q(x, y)+B q(x, f x)+C q(y, f y)+D q(x, f y)+E q(y, f x), \\
& q(f y, f x) \preccurlyeq A q(y, x)+B q(f x, x)+C q(f y, y)+D q(f y, x)+E q(f x, y)
\end{aligned}
$$

for all $x, y \in X$, where $A, B, C, D, E$ are nonnegative constants such that $A+B+C+$ $2 D+2 E<1$. Then $f$ has a fixed point in $X$. If $f u=u$, then $q(u, u)=\theta$.

Proof Let $x_{0} \in X$ be arbitrary and form the sequence $\left\{x_{n}\right\}$ with $x_{n}=f^{n} x_{0}$. In order to prove that it is a Cauchy sequence, put $x=x_{n}$ and $y=x_{n-1}$ in (3.1) to get

$$
\begin{gathered}
q\left(x_{n+1}, x_{n}\right) \preccurlyeq A q\left(x_{n}, x_{n-1}\right)+B q\left(x_{n}, x_{n+1}\right)+C q\left(x_{n-1}, x_{n}\right) \\
+D q\left(x_{n}, x_{n}\right)+E q\left(x_{n-1}, x_{n+1}\right) \\
\preccurlyeq A q\left(x_{n}, x_{n-1}\right)+(B+D+E) q\left(x_{n}, x_{n+1}\right) \\
+(C+E) q\left(x_{n-1}, x_{n}\right)+D q\left(x_{n+1}, x_{n}\right) .
\end{gathered}
$$

Similarly, putting $y=x_{n-1}$ and $x=x_{n}$ in (3.2), one obtains

$$
\begin{gathered}
q\left(x_{n}, x_{n+1}\right) \preccurlyeq A q\left(x_{n-1}, x_{n}\right)+B q\left(x_{n+1}, x_{n}\right)+C q\left(x_{n}, x_{n-1}\right) \\
+D q\left(x_{n}, x_{n}\right)+E q\left(x_{n+1}, x_{n-1}\right) \\
\preccurlyeq A q\left(x_{n-1}, x_{n}\right)+(B+D+E) q\left(x_{n+1}, x_{n}\right) \\
+(C+E) q\left(x_{n}, x_{n-1}\right)+D q\left(x_{n}, x_{n+1}\right) .
\end{gathered}
$$

Denote $u_{n}=q\left(x_{n+1}, x_{n}\right)+q\left(x_{n}, x_{n+1}\right)$. Adding up (3.3) and (3.4), we get that

$$
u_{n} \preccurlyeq(A+C+E) u_{n-1}+(B+2 D+E) u_{n},
$$

i.e. $u_{n} \leqslant h u_{n}-1$ with

$$
0 \leq h=\frac{A+C+E}{1-B-2 D-E}<1,
$$

since $A+B+C+2 D+2 E<1$ and, e.g., $A+C+E>0$. 
By induction, $u_{n} \leqslant h^{n} u_{0}$ and $q\left(x_{n}, x_{n+1}\right) \leqslant u_{n} \leqslant h^{n}\left(q\left(x_{1}, x_{0}\right)+q\left(x_{0}, x_{1}\right)\right)$. In the usual way, it follows that

$$
q\left(x_{n}, x_{m}\right) \preccurlyeq \frac{h^{n}}{1-h}\left(q\left(x_{1}, x_{0}\right)+q\left(x_{0}, x_{1}\right)\right)=v_{n}
$$

for $m>n$, where $\left\{v_{n}\right\}$ is a $c$-sequence. Lemma 1.(3) implies that $\left\{x_{n}\right\}$ is a Cauchy sequence in $X$ and, since $X$ is complete, $x_{n} \rightarrow x^{*} \in X(n \rightarrow \infty)$. Continuity of $f$ implies that $x_{n+1}=f x_{n} \rightarrow f x^{*}$, and since the limit of a sequence in tvs-cone metric space in unique, we get that $f x^{*}=x^{*}$.

Suppose that $f u=u$. Then, (3.1) implies that

$$
\begin{aligned}
q(u, u) & =q(f u, f u) \preccurlyeq A q(u, u)+B q(u, u)+C q(u, u)+D q(u, u)+E q(u, u) \\
& =(A+B+C+D+E) q(u, u),
\end{aligned}
$$

which is, by property $\left(\mathrm{p}_{5}\right)$ and $A+B+C+D+E<A+B+C+2 D+2 E<1$, possible only if $q(u, u)=\theta$.

Some special cases of the previous theorem, for example Banach-type and Kannantype fixed point results, need only one condition:

$$
q(f x, f y) \preccurlyeq \lambda q(x, y), \quad \lambda \in[0,1),
$$

and

$$
q(f x, f y) \preccurlyeq \lambda(q(x, f x)+q(y, f y)), \quad \lambda \in[0,1 / 2),
$$

respectively.

Remark 1 If the underlying cone $P$ of the given tvs-cone metric space $(X, d)$ is normal (and, hence, this space is a cone metric space in the sense of [1], see Theorem 2.1), then continuity of $f$ in Theorem 2 can be replaced by the condition

$$
\inf \{\|q(x, y)\|+\|q(x, f x)\|: x \in X\}>0 \text { for all } y \in X \text { with } y \neq f y .
$$

It may be of interest to note that in this case, property (q3) of $c$-distance has to be used in the course of the proof (see, e.g., the respective procedure in ordered cone metric spaces in [19]), while in our case (when $f$ is continuous), this property is not needed.

The next is a result including two mappings and the existence of their common fixed point.

Theorem 3 Let $(X, d)$ be a complete tvs-cone metric space and let $q$ be a c-distance on $X$. Suppose that continuous self-maps $f, g: X \rightarrow X$ satisfy the following two conditions:

$$
\begin{aligned}
& q(f x, g y) \preccurlyeq A q(x, y)+B[q(x, f x)+q(y, g y)]+D[q(x, g y)+q(y, f x)], \\
& q(g y, f x) \preccurlyeq A q(y, x)+B[q(f x, x)+q(g y, y)]+D[q(g y, x)+q(f x, y)]
\end{aligned}
$$

for all $x, y \in X$, where $A, B, D$ are nonnegative constants, such that $A+2 B+4 D<1$. Then $f$ and $g$ have a common fixed point in $X$. If $f u=g u=u$, then $q(u, u)=\theta$.

Proof Let $x_{0} \in X$ be arbitrary and form the sequence $\left\{x_{n}\right\}$ such that $x_{2 n+1}=f x_{2 n}$ and $x_{2 n+2}=g x_{2 n+1}$ for $n \geq 0$. Denote $u_{n}=q\left(x_{2 n}, x_{2 n+1}\right)+q\left(x_{2 n+1}, x_{2 n}\right)$ and $v_{n}=q\left(x_{2 n+1}, x_{2 n+2}\right)$ $+q\left(x_{2 n+2}, x_{2 n+1}\right)$. 
Putting $x=x_{2 n+2}, y=x_{2 n+1}$ in (3.5) we obtain that

$$
\begin{gathered}
q\left(x_{2 n+3}, x_{2 n+2}\right) \preccurlyeq A q\left(x_{2 n+2}, x_{2 n+1}\right)+B\left[q\left(x_{2 n+2}, x_{2 n+3}\right)+q\left(x_{2 n+1}, x_{2 n+2}\right)\right] \\
\quad+D\left[q\left(x_{2 n+2}, x_{2 n+2}\right)+q\left(x_{2 n+1}, x_{2 n+3}\right)\right] \\
\preccurlyeq A q\left(x_{2 n+2}, x_{2 n+1}\right)+(B+2 D) q\left(x_{2 n+2}, x_{2 n+3}\right) \\
+(B+D) q\left(x_{2 n+1}, x_{2 n+2}\right)+D q\left(x_{2 n+3}, x_{2 n+2}\right) .
\end{gathered}
$$

Similarly, putting the same values for $x, y$ in (3.6), we get

$$
\begin{gathered}
q\left(x_{2 n+2}, x_{2 n+3}\right) \preccurlyeq A q\left(x_{2 n+1}, x_{2 n+2}\right)+B\left[q\left(x_{2 n+3}, x_{2 n+2}\right)+q\left(x_{2 n+2}, x_{2 n+1}\right)\right] \\
+D\left[q\left(x_{2 n+2}, x_{2 n+2}\right)+q\left(x_{2 n+3}, x_{2 n+1}\right)\right] \\
\preccurlyeq A q\left(x_{2 n+1}, x_{2 n+2}\right)+(B+2 D) q\left(x_{2 n+3}, x_{2 n+2}\right) \\
+(B+D) q\left(x_{2 n+2}, x_{2 n+1}\right)+D q\left(x_{2 n+2}, x_{2 n+3}\right) .
\end{gathered}
$$

It follows by adding up (3.7) and (3.8) that

$$
u_{n+1} \preccurlyeq(A+B+D) v_{n}+(B+3 D) u_{n+1},
$$

i.e.,

$$
u_{n+1} \preccurlyeq h v_{n}, \quad n \in \mathbb{N},
$$

where $0<h=\frac{A+B+D}{1-B-3 D}<1$, since $A+B+D>0$ and $A+2 B+4 D<1$.

By a similar procedure, starting with $x=x_{2 n}$ and $y=x_{2 n+1}$, one can get

$$
v_{n} \preccurlyeq h u_{n}, \quad n \in \mathbb{N} .
$$

Combining the last two inequalities, it follows that

$$
u_{n+1} \preccurlyeq h^{2} u_{n} \text { and } v_{n} \preccurlyeq h^{2} v_{n-1} \text { ， }
$$

and we get that $\left\{u_{n}\right\}$ and $\left\{v_{n}\right\}$ are $c$-sequences. We have that $q\left(x_{2 n}, x_{2 n+1}\right) \leqslant u_{n}, q\left(x_{2 n}\right.$ $\left.{ }_{+1}, x_{2 n+2}\right) \leqslant v_{n}$ and it follows that $q\left(x_{n}, x_{n+1}\right) \leqslant u_{n}+v_{n}$, where $u_{n}+v_{n}$ is a $c$-sequence. Using Lemma 1.(3), we obtain that $\left\{x_{n}\right\}$ is a Cauchy sequence in $X$. Hence, $x_{n} \rightarrow x^{*} \in$ $X(n \rightarrow \infty)$. Since $f$ and $g$ are continuous, it easily follows from the definition of $\left\{x_{n}\right\}$ that $f x^{*}=g x^{*}=x^{*}$.

Thus, mappings $f$ and $g$ have a common fixed point. Suppose that $u \in X$ is any point satisfying $f u=g u=u$. Then, (3.5) implies that

$$
\begin{aligned}
q(u, u) & =q(f u, g u) \preccurlyeq A q(u, u)+B[q(u, u)+q(u, u)]+D[q(u, u)+q(u, u)] \\
& =(A+2 B+2 D) q(u, u)
\end{aligned}
$$

and, since $0<A+2 B+2 D<A+2 B+4 D<1$, property $\left(\mathrm{p}_{5}\right)$ implies that $q(u, u)=$ $\theta$.

As corollaries, we obtain, for example, common fixed point result for self-maps $f$ and $g$ satisfying

$$
q(f x, g y) \preccurlyeq A q(x, y), \quad q(g y, f x) \preccurlyeq A q(y, x), \quad 0<A<1,
$$

or for a self-map $f$ satisfying

$$
\begin{aligned}
& q\left(f^{n} x, f^{m} y\right) \preccurlyeq A q(x, y)+B\left[q\left(x, f^{n} x\right)+q\left(y, f^{m} y\right)\right]+D\left[q\left(x, f^{m} y\right)+q\left(y, f^{n} x\right)\right], \\
& q\left(f^{m} y, f^{n} x\right) \preccurlyeq A q(y, x)+B\left[q\left(f^{n} x, x\right)+q\left(f^{m} y, y\right)\right]+D\left[q\left(f^{m} y, x\right)+q\left(f^{n} x, y\right)\right],
\end{aligned}
$$

where $m, n \in \mathbb{N}, A+2 B+4 D<1$. 
Remark 2 Similarly as in Remark 1, we note that if the cone $P$ is normal, then continuity of mappings $f$ and $g$ in Theorem 3 can be replaced by conditions

$$
\begin{aligned}
& \inf \{\|q(x, y)\|+\|q(x, f x)\|: x \in X\}>0 \text { for all } y \in X \text { with } y \neq f y, \\
& \inf \{\|q(x, y)\|+\|q(x, g x)\|: x \in X\}>0 \text { for all } y \in X \text { with } y \neq g y .
\end{aligned}
$$

Example 4 Let $E=\mathbb{R}$ and $P=[0,+\infty)$. Let $X=[0,+\infty), d(x, y)=|x-y|$ and define $q$ $(x, y)=x$. It is easy to check that $q$ is a $c$-distance on a cone metric space $(X, d)$.

Take functions $f, g: X \rightarrow X$ defined by $f x=\frac{x}{4}$ and $g x=\frac{x}{2}$. If $x=5, y=\frac{15}{2}$, then $d(f x, g y)=d\left(\frac{5}{4}, \frac{15}{4}\right)=\frac{5}{2}$ and $d(x, y)=d\left(5, \frac{15}{2}\right)=\frac{5}{2}$. Hence, there is no $A \in(0,1)$ (and hence no triple $(A, B, D))$ such that $d(f x, g y) \leq A d(x, y)$ for each $x, y \in[0,+\infty)$, i.e., the existence of a common fixed point of $f$ and $g$ cannot be deduced from the well-known metric version of Theorem 3.

However, conditions of the $c$-distance version (Theorem 3 ) are satisfied. Indeed, take arbitrary $A, \frac{1}{2} \leq A<1$ and $B=D=0$. Then, for each $x, y \in[0,+\infty)$, $q(f x, g y)=f x=\frac{x}{4} \leq A x=A q(x, y)$ and $q(g y, f x)=g y=\frac{\gamma}{2} \leq A y=A q(y, x)$ (see (3.9)). Note that $f$ and $g$ have a (trivial) common fixed point $u=0$ and that $q(u, u)=q(0,0)=0$.

This example can be easily modified to the tvs-cone metric case. It is enough to define tvs-cone metric on $X$ by $d(x, y)(t)=|x-y| \phi(t)$ with fixed $\phi \in P=\{f \in C[0,1]$ : $f(t) \geq 0$ for $t \in[0,1]\}$ and take $c$-distance $q_{1}(x, y)(t)=x \cdot \phi(t)$ (see Example 3).

\subsection{Mappings without periodic points}

The first part of the following result was given with an incorrect proof in [20] (using lim inf which may not be defined in the case of an arbitrary cone metric space).

Recall that a map $f: X \rightarrow X$ is said to have property $(P)$ if it satisfies $F(f)=F\left(f^{p}\right)$ for each $n \in \mathbb{N}$, where $F(f)$ stands for the set of all fixed points of $f$ [22].

Theorem 4 Let $(X, d)$ be a tvs-cone metric space and $q: X \times X \rightarrow E$ be a c-distance on $X$. Suppose that a continuous self-map $f: X \rightarrow X$ satisfies

$$
q\left(f x, f^{2} x\right) \preccurlyeq \lambda q(x, f x)
$$

for some $\lambda \in(0,1)$ and each $x \in X$. Then:

1. $f$ has a fixed point and if $f u=u$, then $q(u, u)=\theta$;

2. $f$ has property $(P)$.

Proof (1) Let $x_{0} \in X$ and $x_{n+1}=f x_{n}, n \geq 0$. If $x_{n_{0}}+1=x_{n_{0}}$ for some $n_{0} \in \mathbb{N}_{0}$, then $x_{n_{0}}$ is a fixed point of $f$. Otherwise, we get from (3.10) that

$$
\begin{aligned}
q\left(x_{n}, x_{n+1}\right) & =q\left(f x_{n-1}, f^{2} x_{n-1}\right) \preccurlyeq \lambda q\left(x_{n-1}, f x_{n-1}\right)=\lambda q\left(f x_{n-2}, f^{2} x_{n-2}\right) \\
& \preccurlyeq \lambda^{2} q\left(x_{n-2}, f x_{n-2}\right) \preccurlyeq \cdots \preccurlyeq \lambda^{n} q\left(x_{0}, x_{1}\right) .
\end{aligned}
$$

Using Lemma 1.(3) again, one obtains that $\left\{x_{n}\right\}$ is a Cauchy sequence in $X$.

Hence, $x_{n} \rightarrow x^{*}$, and continuity of $f$ implies that $x_{n+1}=f x_{n} \rightarrow f x^{*}$ and $f x^{*}=x^{*}$.

(2) Obviously, $F(f) \subseteq F\left(f^{n}\right)$ for each $n \in \mathbb{N}$. Let $u \in F\left(f^{n}\right)$, i.e., $f^{n} u=u$. Then, (3.10) implies that

$$
\begin{aligned}
q(u, f u) & =q\left(f f^{n-1} u, f^{2} f^{n-1} u\right) \preccurlyeq \lambda q\left(f^{n-1} u, f^{n} u\right)=\lambda q\left(f f^{n-2} u, f^{2} f^{n-2} u\right) \\
& \preccurlyeq \lambda^{2} q\left(f^{n-2} u, f^{n-1} u\right) \preccurlyeq \cdots \preccurlyeq \lambda^{n} q(u, f u) .
\end{aligned}
$$

By property $\left(\mathrm{p}_{5}\right)$, it follows that $q(u, f u)=\theta$. 
Now, for arbitrary $k \in\{1,2, \ldots, n\}$, we have that $q\left(f^{k} u, f^{k+1} \mathrm{u}\right) \leqslant \lambda^{k} q(u, f u) \theta$ and so $q$ $\left(f^{k} u, f^{k+1} u\right)=\theta$. It follows that $q\left(u, f^{2} u\right) \leqslant q(u, f u)+q\left(f u, f^{2} u\right)=\theta$, i.e., $q\left(u, f^{2} u\right)=\theta$ and, similarly,

$$
q(u, f u)=q\left(u, f^{2} u\right)=q\left(u, f^{3} u\right)=\cdots=q\left(u, f^{n} u\right)=\theta .
$$

From $q(u, f u)=\theta=q\left(u, f^{n} u\right)=q(u, u)$ and Lemma 1.(1), we conclude that $f u=u$, i. e., $u \in F(f)$.

Another way to obtain property $(P)$ is the following.

Theorem 5 Let $(X, d)$ be a tvs-cone metric space and $q: X \times X \rightarrow E$ be a c-distance on $X$. Suppose that a continuous self-map $f: X \rightarrow X$ satisfies

$$
q\left(f x, f^{2} x\right)+q\left(f^{2} x, f x\right) \preccurlyeq \lambda[q(x, f x)+q(f x, x)]
$$

for some $\lambda \in(0,1)$ and each $x \in X$. Then $f$ has property $(P)$.

Proof Denote $z_{1}(x)=q(x, f x)+q(f x, x)$ and $z_{2}(x)=q\left(f x, f^{2} x\right)+q\left(f^{2} x, f x\right)$.

Then, the given condition is written as $z_{2}(x) \leqslant \lambda z_{1}(x)$ for each $x \in X$. Suppose that $f^{n} u=u$. Then,

$$
\begin{aligned}
z_{1}(u) & =q(u, f u)+q(f u, u)=q\left(f^{n} u, f f^{n} u\right)+q\left(f f^{n} u, f^{n} u\right)=z_{2}\left(f^{n-1} u\right) \\
& \preccurlyeq \lambda z_{1}\left(f^{n-1} u\right)=\lambda z_{2}\left(f^{n-2} u\right) \preccurlyeq \lambda^{2} z_{1}\left(f^{n-2} u\right) \preccurlyeq \cdots \preccurlyeq \lambda^{n} z_{1}(u) .
\end{aligned}
$$

Since $0<\lambda^{n}<1$, property $\left(\mathrm{p}_{5}\right)$ implies that $z_{1}(u)=q(u, f u)+q(f u, u)=\theta$. Again, the triangle inequality (q2) implies that $q(u, u)=q(f u, f u)=\theta$, and by Lemma 1.(1), we get that $f u=u$.

Corollary 1 Let $q$ be a c-distance on a tvs-cone metric space $(X, d)$ and let $f: X \rightarrow X$ be continuous and such that for some nonnegative $A, B, C, D, E$ such that $A+B+C+$ $2 D+2 E<1$, inequalities (3.1) and (3.2) hold for all $x, y \in X$. Then $f$ has property $(P)$.

Proof Putting $x=x$ and $y=f x$ in conditions (3.1) and (3.2) leads to the following inequalities:

$$
\begin{aligned}
& q\left(f x, f^{2} x\right) \preccurlyeq(A+B+D) q(x, f x)+(C+D+E) q\left(f x, f^{2} x\right)+E q\left(f^{2} x, f x\right), \\
& q\left(f^{2} x, f x\right) \preccurlyeq(A+B+D) q(f x, x)+(C+D+E) q\left(f^{2} x, f x\right)+E q\left(f x, f^{2} x\right) .
\end{aligned}
$$

Adding up, one obtains inequality (3.11) with $0<\lambda=\frac{A+B+D}{1-C-D-2 E}<1$, since $A$ $+B+C+2 D+2 E<1$.

Similar results concerning property $(Q)$ of two self-mappings $f$ and $g$ (i.e., property that $F(f) \cap F(g)=F\left(f^{n}\right) \cap F\left(g^{n}\right)$ for each $\left.n \in \mathbb{N}\right)$ can be obtained.

\section{Acknowledgements}

The authors thank the referees for their valuable comments that helped to improve the text. The authors are thankful to the Ministry of Science and Technological Development of Serbia.

\section{Competing interests}

The authors declare that they have no competing interests.

\section{Authors' contributions}

All authors contributed equally and significantly in writing this paper. All authors read and approved the final manuscript.

\section{Author details}

${ }^{1}$ Managment for Logistics (J-4) GŠ VS, Beograd, Serbia ${ }^{2}$ Faculty of Organizational Sciences, University of Belgrade, Jove Ilića 154, 11000 Beograd, Serbia ${ }^{3}$ Faculty of Mathematics, University of Belgrade, Studentski trg 16, 11000 Beograd, Serbia ${ }^{4}$ Faculty of Mechanical Engineering, University of Belgrade, Kraljice Marije 16, 11120 Beograd, Serbia 


\section{References}

1. Huang, LG, Zhang, X: Cone metric spaces and fixed point theorems of contractive mappings. J Math Anal Appl. 332(2), 1468-1476 (2007). doi:10.1016/j.jmaa.2005.03.087

2. Vandergraft, JS: Newton's method for convex operators in partially ordered spaces. SIAM J Num Anal. 4, 406-432 (1967). doi:10.1137/0704037

3. Krasnoseliski, MA, Zabrejko, PP: Geometrical Methods in Nonlinear Analysis. Springer, Berlin (1984)

4. Zabrejko, PP: K-metric and K-normed linear spaces, survey. Collect Math. 48, 825-859 (1997)

5. Beg, l, Azam, A, Arshad, M: Common fixed points for maps on topological vector space valued cone metric spaces. Intern J Math Math Sci (Article ID 560264), 8 (2009).

6. Wei-Shih, Du: A note on cone metric fixed point theory and its equivalence. Nonlinear Anal. 72, 2259-2261 (2010). doi:10.1016/.na.2009.10.026

7. Kadelburg, Z, Radenović, S, Rakočević, V: Topological vector space valued cone metric spaces and fixed point theorems. Fixed Point Theory Appl 2010, (Article ID 170253), 18 (2010).

8. Abdeljawad, Th, Rezapour, Sh: On some topological concepts of TVS-cone metric spaces and fixed point theory remarks. arXiv, 1102.1419v1[math.GN]

9. Abdeljawad, Th, Karapinar, E: A gap in the paper "A note on cone metric fixed point theory and its equivalence. Nonlinear Anal 72, 2259-2261 (2010). Gazi Univ. J. Sci. 24(2), 233-234 (2011). doi:10.1016/..na.2009.10.026

10. Abdeljawad, Th, Karapinar, E: A common fixed point theorem of Gregus type on convex cone metric spaces. J Comput Anal Appl. 13(4), 609-621 (2011)

11. Arandjelović, ID, Kečkić, DJ: On nonlinear qausi-contractions on TVS-valued cone metric spaces. Appl Math Lett. 24 1209-1213 (2011). doi:10.1016/j.aml.2011.02.010

12. Karapinar, E, Yuksel, U: On common fixed point theorem without commuting conditions in TVS-cone metric spaces. J Comput Anal Appl. 13, 1123-1131 (2011)

13. Simić, Su: A note on Stone's, Baire's, Ky Fan's and Dugundji's theorem in tvs-cone metric spaces. Appl Math Lett. 24, 999-1002 (2011). doi:10.1016/j.aml.2011.01.014

14. Janković, S, Kadelburg, Z, Radenović, S: On cone metric spaces, a survey. Nonlinear Anal. 74, 2591-2601 (2011). doi:10.1016/j.na.2010.12.014

15. Kada, O, Suzuki, T, Takahashi, W: Nonconvex minimization theorems and fixed point theorems in complete metric spaces. Math Japonica. 44, 381-391 (1996)

16. Abbas, M, llić, D, Ali Khan, M: Coupled coincidence point and coupled common fixed point theorems in partially ordered metric spaces with w-distance. Fixed Point Theory Appl 2010, (Article ID 134897), 11 (2010).

17. Ilić, D, Rakočević, V: Common fixed point for maps with w-distance. Appl Math Comput. 199, 599-610 (2008). doi:10.1016/jamc.2007.10.016

18. Razani, A, Nezhad, ZM, Boujary, M: A fixed point theorem for w-distance. Appl Sci. 11, 114-117 (2009)

19. Cho, YJ, Saadati, R, Wang, Sh: Common fixed point theorems on generalized distance in ordered cone metric spaces. Comput Math Appl. 61, 1254-1260 (2011). doi:10.1016/j.camwa.2011.01.004

20. Lakzian, H, Arabyani, F: Some fixed point theorems in cone metric spaces with w-distance. Inter J Math Anal. 3(22), 1081-1086 (2009)

21. Abdeljawad, Th: Completions of tvs-cone metric spaces and some fixed point theorems. Gazi Univ J Sci. 24(2), 235-240 (2011)

22. Jeong, GS, Rhoades, BE: Maps for which $F(T)=F\left(T^{n}\right)$. Fixed Point Theory Appl. 6, 87-131 (2005)

doi:10.1186/1687-1812-2011-29

Cite this article as: Đorđevic et al:: Fixed point results under c-distance in tvs-cone metric spaces. Fixed Point Theory and Applications 2011 2011:29.

\section{Submit your manuscript to a SpringerOpen ${ }^{\circ}$ journal and benefit from:}

- Convenient online submission

- Rigorous peer review

- Immediate publication on acceptance

- Open access: articles freely available online

- High visibility within the field

- Retaining the copyright to your article

Submit your next manuscript at $\boldsymbol{s p r i n g e r o p e n . c o m ~}$ 